\title{
Prediction of endotracheal tube size for pediatric patients from the epiphysis diameter of radius
}

\author{
Hee Young Kim, Ji Hyun Cheon, Seung Hoon Baek, Kyung Hoon Kim, and \\ Tae Kyun Kim \\ Department of Anesthesia and Pain Medicine, Pusan National University Yangsan Hospital and School of Medicine, \\ Yangsan, Korea
}

\begin{abstract}
Background: Using a too big or a too small size of an endotracheal tube in pediatric patients would result in tracheal injury or insufficient ventilation. Determining the appropriate endotracheal tube size is important because using an inappropriate size can cause complications. This study was performed to predict the appropriate endotracheal tube size by measuring the transverse diameter of the epiphysis of the distal radius under the assumption that the growth rates of cartilages in the entire body are close to each other.

Methods: Fifty-eight children aged 3 to 10 years who required general anesthesia were intubated with an uncuffed endotracheal tube. The tube size was considered to be appropriate when leaks occurred at inspiratory peak pressures between 10 to $25 \mathrm{mmHg}$. The transverse diameters of the epiphysis were measured with an ultra-sonogram at the distal radius and the proximal phalanx of the third finger and the fifth finger. Correlations and prediction probabilities of measurements were evaluated. The number needed to harm $(\mathrm{NNH})$, which indicates the number of patients who need to be intubated for one patient who needs tube exchange, was investigated.

Results: The Spearman's correlation coefficient between the endotracheal tube size and the epiphysis of the distal radius was 0.814 , which was the biggest coefficient. For epiphysis of the proximal phalanx of the third finger and the fifth finger, the correlation coefficient was 0.704 and 0.701 , respectively. If the Cole's formula was applied for selection of the tube size, the NNH would be 7.

Conclusions: The appropriate endotracheal tube size could be predicted by means of the epiphyseal transverse diameter of the distal radius rather than the circumference measurements of the phalanx.
\end{abstract}

Key Words: Epiphyses, Intratracheal intubation, Pediatrics, Radius, Ultrasonography.

Corresponding author: Tae Kyun Kim, M.D., Ph.D.

Department of Anesthesia and Pain Medicine, Pusan National University Yangsan Hospital and School of Medicine, 20, Geumo-ro, Mulgeum-eup, Yangsan 50612, Korea

Tel: 82-55-360-2129, Fax: 82-55-360-2149

Email: anesktk@pusan.ac.kr

ORCID: http://orcid.org/0000-0002-4790-896X

Received: June 10, 2016.

Revised: July 25, 2016 (1st); August 10, 2016 (2nd); August 12, 2016 (3rd). Accepted: August 30, 2016.

Korean J Anesthesiol 2017 February 70(1): 52-57

https://doi.org/10.4097/kjae.2017.70.1.52

\section{Introduction}

In pediatric anesthesia environment, selection of the appropriate size of an endotracheal tube (ETT) is one of the main issues because an inappropriately large or small size of the ETT causes tracheal edema or leakage. Airway edema induced by multiple trials of intubation might result in hypoxemia in the end [1]. In order to prevent the complications of multiple trials of intubation, many methods which can precisely predict the appropriate size of an ETT have been suggested.

Some of the anesthesiologists used to choose the ETT size based on their own experience, or other anesthesiologists would

(c) This is an open-access article distributed under the terms of the Creative Commons Attribution Non-Commercial License (http://creativecommons.org/ licenses/by-nc/4.0/), which permits unrestricted non-commercial use, distribution, and reproduction in any medium, provided the original work is properly cited. 
select the appropriate ETT size by calculating formulae which are based on the children's demographic data such as weight, height or age. Due to the variable rate of child development, demographic data-based prediction of the ETT size has been criticized. Nevertheless, the Cole's equation [2] is still considered as one of the prevalent methods in spite of its poor prediction power.

Many studies on selection of the appropriate size of an ETT in pediatric patients have been reported, and there have been many debates regarding which formula or method is superior to the other formulae or methods. The need for making a quick decision on the ETT size has led some of the anesthesiologists to use intuitive methods such as using the transverse diameter or width of the child's fifth fingernail to select the size of an ETT [3]. However, a few reports have stated that measuring the fingertip transverse diameter or width to predict the ETT size is unreliable $[4,5]$.

Nevertheless, the claim that some part of body growth would be associated with tracheal size still appears valid because child growth is accomplished with overall growth of the body. Instead of measurements of the fingertip transverse diameter or width, measurements of bony cartilage growth of the hand are worthy of attention as a surrogate marker of tracheal diameter because the bone and cartilage growth of the body are supposed to be related to each other and bone age for the hand is generally accepted as the indicator of growth.

We hypothesized that the epiphyseal transverse diameter of the hand would be closely correlated with the growth of tracheal diameter in pediatric patients, and this study was performed to identify the relationship between the appropriate ETT size and epiphyseal growth of the hand and finally to create a highly predictable formula which can be applied for selecting the appropriate ETT size.

\section{Materials and Methods}

This study was approved by the hospital Institutional Review Board, and written informed consent was obtained from the parents of children. Children with the American Society of Anesthesiologists physical status classification I, aged 3 to 10 years, and who required general anesthesia were scheduled for investigation. Children with conditions that are known or suspected to have laryngeal or tracheal pathology were excluded. Children who had musculoskeletal disorders, congenital developmental disorders, malformation of the hand or extremities were also excluded.

General anesthesia was induced with intravenous thiopental sodium $5 \mathrm{mg} / \mathrm{kg}$, and it was maintained with sevoflurane. Intravenous rocuronium bromide $0.6 \mathrm{mg} / \mathrm{kg}$ was injected for muscle relaxation. After complete muscle relaxation was achieved, the first trial of intubation was carried out using an uncuffed endotracheal tube, which was selected according to the agebased formula (internal diameter [ID] in $\mathrm{mm}=$ [age in years + 16]/4) suggested by Cole [2]. Polyvinyl chloride uncuffed ETTs (Mallinckrodt, Athlone, Ireland), ranging in size from ID 4.0 to $7.0 \mathrm{~mm}$ with a step size of $0.5 \mathrm{~mm}$, were prepared.

Immediately after placement of the tube in the trachea, air leakage was monitored by performing auscultation on the skin over the larynx with a stethoscope. If a leak occurred at an airway pressure of less than $10 \mathrm{cmH}_{2} \mathrm{O}$ when the lungs were inflated, the tube size used was considered to be small to fit into the tracheal lumen. In that case, the tube was changed to a tube having a one step larger size. If there was no audible leak above an airway pressure of $25 \mathrm{cmH}_{2} \mathrm{O}$, the tube was considered to be large and it was changed to a tube having a step smaller size. This process was continued until the leak occurred only at an airway pressure between 10 to $25 \mathrm{cmH}_{2} \mathrm{O}$.

After induction, several parts of the hand were examined
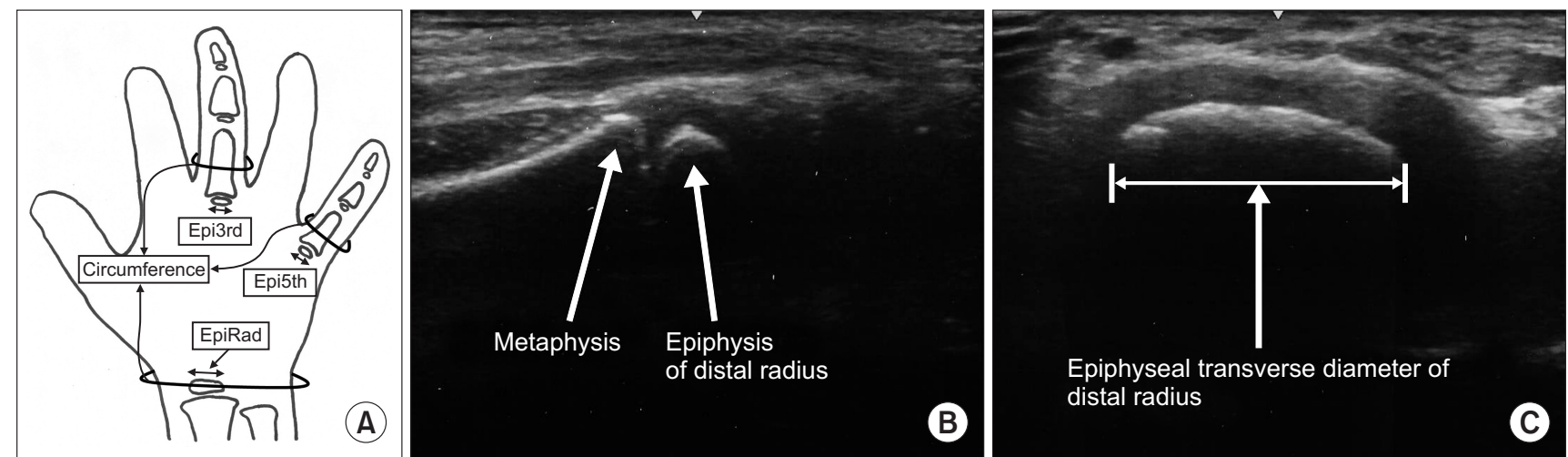

Fig. 1. Epiphyseal transverse diameter of the distal radius (EpiRad), the 3rd proximal phalanx (Epi3rd) and the 5th (Epi5th) proximal phalanx and circumference of the corresponding parts were measured (A). Ultrasonography shows longitudinal (B) and transverse (C) images of the epiphysis of the distal radius. 
by another physician using an ultrasonography system with a 12L-RS linear probe (LOGIQ e, GE Healthcare, Milwaukee, WI, USA). The physician was blinded to the determined ETT size. The epiphyseal transverse diameter of the distal radius, the $3 \mathrm{rd}$ proximal phalanx, and the 5th proximal phalanx was estimated (Fig. 1). Circumferences of the wrist, the 3rd proximal phalanx, the 5th proximal phalanx, and the neck were measured. The neck circumference was measured at the level of the cricoid ring.

The primary outcome of this study was to create a reliable formula for selecting the appropriate ETT size with best correlated measurement. The minimum required sample size for linear regression was determined as 52.3 , given the desired significance level of 0.05 , the desired statistical power level of 0.8 and the anticipated effect size of 0.15 , according to the Cohen's small effect size. Considering a $10 \%$ dropout rate, 58 children were scheduled for investigation. Regression model was built based on the measurement which resulted in best correlation and prediction performance. Spearman's correlation analysis was performed to determine the relationship between the applied ETT sizes and ultrasonographic or circumferential measurements of the hand part. Prediction performance was calculated for determining which measurement could be the best indicator of the ETT size. For the prediction performance measurement, the prediction probability $(\mathrm{Pk})$ was calculated as described by Smith et al. [6] and programmed by Jordan et al. [7]. Normality assumption of the residual error was investigated with the QQ plot and tested with the Shapiro-Wilk test. Constant variance assumption of the residual error was investigated with the residuals versus predicted values plot and tested with the BreuschPagan test. Assumption tests were performed with use of $\mathrm{R}$ statistical software (version 3.3.0; the R Foundation for Statistical Computing, Vienna, Austria).

The number needed to harm (NNH), which indicates the minimum number of patients who need to be intubated until one patient who needs tube exchange is obtained, was evaluated. The term 'harm' in the NNH was defined as multiple intubations in a child. The NNH value was evaluated for Cole's [2], Eck's [8], and Circ5th's formulae in comparison with the EpiRad formula. $\mathrm{NNH}$ values were calculated from the inverse of the absolute difference in ETT exchange incidences as follows:

$$
\mathrm{NNH}=1 / \mathrm{ARI}=1 /\left(\mathrm{R}_{\mathrm{t}}-\mathrm{R}_{\mathrm{c}}\right),
$$

Table 1. Patient Demographic Characteristics

\begin{tabular}{lc}
\hline & Total $(\mathrm{n}=58)$ \\
\hline Age (months) & $70 \pm 21(36,120)$ \\
Sex $(\mathrm{M} / \mathrm{F})$ & $37 / 21$ \\
Height $(\mathrm{cm})$ & $110 \pm 19(74,147)$ \\
Weight $(\mathrm{kg})$ & $22 \pm 7(13,46)$ \\
\hline
\end{tabular}

Data are expressed as mean \pm SD (range) or number. where absolute risk increase (ARI) is absolute risk increase, the so-called attributable risk, $\mathrm{R}_{\mathrm{t}}$ is ETT exchange rate in the treatment group, $\mathrm{R}_{\mathrm{c}}$ is ETT exchange rate in the control group. $\mathrm{NNH} 1$ indicates that every patient who undergoes intubation needs tube exchange.

\section{Results}

The demographic characteristics of children are shown in Table 1. The correlation coefficient between measurements and the selected ETT tube are shown in Table 2. The highest correlation coefficient $(r=0.814)$ was found in the epiphyseal transverse diameter of the distal radius. The second and third highest correlation coefficients were found in the epiphyseal transverse diameter of the $3 r d$ proximal phalanx $(r=0.704)$ and the 5 th proximal phalanx $(r=0.701)$, respectively. However, the circumference of the neck showed the worst correlation coefficient $(r=0.43)$. The circumference of the fifth finger also showed a low correlation coefficient $(r=0.505)$. Generally, the epiphyseal transverse diameter measurements of the distal radius, the $3 \mathrm{rd}$ proximal phalanx, and the 5th proximal phalanx showed higher correlation coefficients than the circumferential measurements at the corresponding part even though there was no significant difference between their correlation coefficients. Prediction probabilities of measurements are also shown in Table 2 with their own confidence interval. Prediction probability of epiphyseal transverse diameter measurements of the distal radius ( $\mathrm{Pk}$ $=0.896$ ) was the most powerful, but the circumference of the neck showed the least prediction probability $(\mathrm{Pk}=0.666)$. However, there was no significant difference between their predictive probabilities.

Regression analysis between the selected appropriate size of the ETT and epiphyseal transverse diameter measurements of the distal radius (EpiRad) showed the following relationship: $\operatorname{ETT}(\mathrm{mm})=3.668(3.276$ to 4.06$)+0.125(0.101$ to 0.149$) \times$

Table 2. Correlation Coefficient (R) and Prediction Probability (Pk)

\begin{tabular}{lcl}
\hline & $\mathrm{R}$ & \multicolumn{1}{c}{$\mathrm{Pk}$} \\
\hline EpiRad & 0.814 & $0.896(0.825,0.944)$ \\
Epi3rd & 0.704 & $0.842(0.739,0.918)$ \\
Epi5th & 0.701 & $0.834(0.738,0.904)$ \\
CircNeck & 0.430 & $0.666(0.567,0.78)$ \\
CircWrst & 0.583 & $0.767(0.704,0.853)$ \\
Circ3rd & 0.558 & $0.747(0.68,0.836)$ \\
Circ5th & 0.505 & $0.731(0.636,0.839)$
\end{tabular}

EpiRad, Epi3rd and Epi5th: epiphysis of the distal radius, proximal phalanx of the 3rd and 5th finger, respectively. CircNeck, CircWrst, Circ3rd and Circ5th: circumference of the neck, wrist, and proximal phalanx of the 3 rd finger and the 5th finger, respectively. Pk values are presented as mean $(95 \% \mathrm{CI})$. 
EpiRad $(\mathrm{mm})\left(\mathrm{R}^{2}=0.66, \mathrm{P}<0.001\right)$. By accepting a very little difference, this formula can be rephrased in a tidy way as follows: ETT $(\mathrm{mm})=(29.5+\operatorname{EpiRad}[\mathrm{mm}]) / 8$. The confidence interval and prediction interval of the regression are presented in Fig. 2. The sample passed the Shapiro-Wilk test for normality assumption ( $\mathrm{P}=0.608)$ and it also passed the Breusch-Pagan test for homoscedasticity assumption $(\mathrm{P}=0.198)$

Values for NNH and ARI are presented in Table 3 for different formulae; Cole's, Eck's and Circ5th. NNH value for Cole's and Eck's formulae was 7, which indicates that seven patients are needed for one patient to undergo tube exchange. For Circ5th formula, the NNH value was 5 . However, the confidence intervals of ARI for Cole's and Eck's formulae did not exclude zero.

\section{Discussion}

The aim of the current investigation was to develop a new prediction model for the appropriate ETT size which would minimize the changing trial of the ETT. There have been many reports which suggest prediction modalities of the ETT size in pediatric patients. Despite being the most widely used formula and having been reported to be reliable for the past several de-

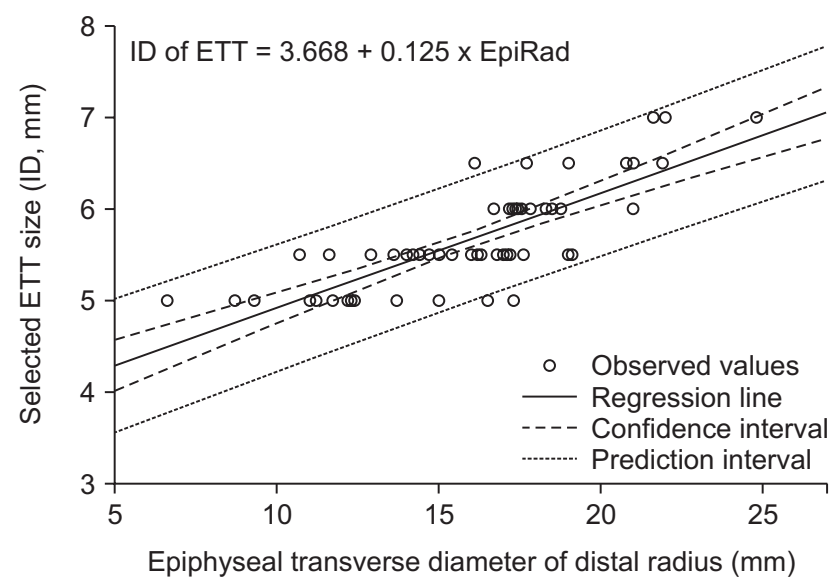

Fig. 2. The plot of regression analysis with both $95 \%$ confidence interval and prediction interval. The plot equation could be rephrased in a simple form as follows: $(29.5+$ EpiRad $) / 8$. EpiRad: transverse diameter of the distal radius, ID: internal diameter, ETT: endotracheal tube. cades, poor predictability of the age-based Cole's formula has been reported [9-11]. Several studies reported that height-based measurements showed reliable prediction rather than age or weight based prediction $[3,12,13]$. However, other studies suggested that age-based prediction might be more accurate than the other formulae $[4,14,15]$. In the midst of such debates, some authors would like to conclude that prediction of the ETT size was best accomplished by performing multivariate regression analysis of the demographic data [8].

Even though demographic data have the advantage that they can be easily obtained, the growth of the trachea would not always be proportional to the demographic data. This is the reason why the methods which predict ETT sizes based on the demographic data have been criticized. From the view point that the tracheal ring is composed of cartilage, the measurement of cartilage growth of the body part, especially the hand, deserves attention as a surrogate marker of cartilage growth of the trachea and the appropriate ETT size. The results of better predictability of the epiphyseal transverse diameter suggest that the measurements of cartilage growth of the body would be more reliable than the circumference of the corresponding part. Even though it is located closest to the trachea, the circumference of the neck is obviously influenced by the muscle and fat, and consequently it showed a coarse correlation with the appropriate ETT size and worse prediction probability for the appropriate ETT size.

There are several reports that measured the tracheal diameter directly by using ultrasonography or radiography [9-11]. With ultrasonography, direct measurement of the diameter of the narrowest subglottic portion might be the best way to predict the appropriate ETT size. However, the narrowest portion was reported to be at the vocal cord and subvocal cord levels in unparalyzed children and it was difficult to visualize these portions because of blurred ultrasonic visualization. Measurement of the diameter via radiography appears to be a good method, but, it might be available for those who had a chest X-ray including the airway. Measurement of the epiphysis of the distal radius with ultrasonography is easy to perform and it is compliable enough in pediatric patients as it can be performed not only at the preoperative evaluation step but also during the induction period in the operating room.

Table 3. Number Needed to Harm (NNH) for Selecting the Appropriate ETT Size

\begin{tabular}{lccc}
\hline Selection formulae & Cole & Eck & Circ5th \\
\hline ARI (\%) $(95 \%$ CI $)$ & $15.5(-2.2,33.3)$ & $14.4(-5,33.7)$ & $20.7(3,38.4)$ \\
NNH & 7 & 7 & 5 \\
\hline
\end{tabular}

Cole: Cole's formula (age +16 ) $/ 4$ [2], Eck: Eck's formula $2.44+$ age $\times 0.1+$ height $\times 0.02+$ weight $\times 0.016$, which was applied to children aged under 7 years [8], Circ5th: based on the circumference of the fifth finger in the current study. ARI: absolute risk increase. NNH: the minimum number of patients who need to be intubated until one patient who needs tube exchange is obtained. The term 'harm' in the NNH was defined as multiple intubations. The NNH was evaluated for each selection formula in comparison with the EpiRad formula. 
The ages of patients included in the current study ranged from three years to ten years. The indicators of bone growth in children are generally based on the appearance of epiphyseal ossification centers and an increase in width of the epiphysis of the hand. The appearance of epiphyseal ossification centers is usually established at the end of the toddling period which is usually until the age of three years. The growth and increase in width of the epiphysis is a matter of concern during the pre-pubertal period, which is usually from the age of three to nine years [16]. In Koreans, the pre-pubertal period would be considered up to the age of 12 years [17].

In clinical fields, use of the uncuffed endotracheal tube is usually not recommended when the child is above 8 years of age and an uncuffed tube larger than size 6 is needed. However, the main aim of this study is to identify the relationship between uncuffed endotracheal tube size and growth of the epiphysis which is hypothesized as a surrogate indicator of tracheal growth. To investigate this relationship adequately in a wide range of epiphyseal growth, age above 8 years and ETT size larger than 6 should be considered as the subjects of interest. There may be several reports that included children whose ages were above 8 years $[5,12,18,19]$.

Even though the ages are equal, in the current study, the transverse diameters of the distal radius and the applied ETT sizes would be different. For example, a three-year-old child whose development status was only $20 \%$ compared to the same age population showed $8.7 \mathrm{~mm}$ of EpiRad and an ETT size 5 was applied. However, another child of the same age whose growth rate was $85 \%$ of the population showed $11.6 \mathrm{~mm}$ of EpiRad and an ETT size 5.5 was applied. Another child aged 73 months and whose height development was less than even 3\% showed 11 $\mathrm{mm}$ of EpiRad, which was smaller than that in the previously mentioned three-year-old child. Consequently, ETT size 5 was applied in that child. Although there were some mismatches between the ETT size and EpiRad, EpiRad showed the best correlation coefficient and prediction probability and it could be considered one of the best explanatory variables for predicting the appropriate ETT size.

$\mathrm{NNH}$ is one of the useful ways of reporting the harm related to the new treatment compared with the standard treatment. The smaller the NNH, the more the possibility of exchange of the ETT tube. The significance of the NNH can be assessed by its confidence interval. However, the confidence interval of $\mathrm{NNH}$, is occasionally confusing; therefore, the confidence interval of ARI was used as a surrogate for the confidence interval of NNH. The ETT exchange incidences were 0.5 and 0.34 for Cole's and EpiRad formulae, respectively, and the value per se appeared to be significantly risky for Cole's formula. Nevertheless, confidence interval of ARI did not exclude zero, which indicates that the increased ETT exchange risk by selection of Cole's for- mula was not very high for someone to state that the increased risk was significant.

A few limitations of the current study should be noted. This method could not be applied in children who had hand deformities or disease related to cartilage or bone growth. It might be difficult to use this method in pediatric patients who are so young that they do not show formation of the epiphysis or are so mature that epiphysis is already capped. If the criteria of the leak test are different, the selected appropriate size of the ETT would be different and the subsequent regression would also be different. There was a report which showed that pressure above 25 $\mathrm{cmH}_{2} \mathrm{O}$ can cause adverse events in children [20]. The remarked sizes of ETTs, which are sold commercially, usually indicate the inner diameter. However, the factor that affects the result of the leak test is actually the outer diameter rather than the inner diameter. The outer diameters of ETTs differ depending on the manufacturer; hence, the results of the leak test would vary depending on the manufacturer of the ETT tube even though an ETT with the same inner diameter is used. No matter how good are the formulae, they would always give wrong predictions. Therefore, preparing an ETT one step larger and smaller than the predicted size is the most important checkpoint before intubation is carried out in pediatric patients.

All of the hospitals cannot afford ultrasonography, and it would be incorrect to state that all of the pediatric cases should undergo epiphysis measurement. We hope that these types of modalities and viewpoints will help the physicians in predicting the appropriate ETT size more precisely. If more investigations about the relationship between the epiphysis and tracheal growth are carried out in the future, even in the field of difficult airways, growth retardation or overgrowth, the modalities used in the current investigation can be considered to help the physicians in selecting the appropriate ETT size in such complicated cases.

In conclusion, measurement of the epiphyseal growth of the hand showed a more close relationship and superior predictability than the circumference of the corresponding part. The epiphyseal transverse diameter of the distal radius measured by an ultrasonogram could be a good predictor of the appropriate ETT size.

\section{Acknowledgments}

This work was supported by a 2-Year Research Grant of Pusan National University.

\section{ORCID}

Hee Young Kim, http://orcid.org/0000-0001-7809-8739

Tae Kyun Kim, http://orcid.org/0000-0002-4790-896X 


\section{References}

1. Cook TM, MacDougall-Davis SR. Complications and failure of airway management. Br J Anaesth 2012; 109 Suppl 1: i68-i85.

2. Cole F. Pediatric formulas for the anesthesiologist. AMA J Dis Child 1957; 94: 672-3.

3. Keep PJ, Manford ML. Endotracheal tube sizes for children. Anaesthesia 1974; 29: 181-5.

4. King BR, Baker MD, Braitman LE, Seidl-Friedman J, Schreiner MS. Endotracheal tube selection in children: a comparison of four methods. Ann Emerg Med 1993; 22: 530-4.

5. van den Berg AA, Mphanza T. Choice of tracheal tube size for children: finger size or age-related formula? Anaesthesia 1997; 52: 701-3.

6. Smith WD, Dutton RC, Smith NT. Measuring the performance of anesthetic depth indicators. Anesthesiology 1996; 84: 38-51.

7. Jordan D, Steiner M, Kochs EF, Schneider G. A program for computing the prediction probability and the related receiver operating characteristic graph. Anesth Analg 2010; 111: 1416-21.

8. Eck JB, De Lisle Dear G, Phillips-Bute BG, Ginsberg B. Prediction of tracheal tube size in children using multiple variables. Paediatr Anaesth 2002; 12: 495-8.

9. Bae JY, Byon HJ, Han SS, Kim HS, Kim JT. Usefulness of ultrasound for selecting a correctly sized uncuffed tracheal tube for paediatric patients. Anaesthesia 2011; 66: 994-8.

10. Park HP, Hwang JW, Lee JH, Nahm FS, Park SH, Oh AY, et al. Predicting the appropriate uncuffed endotracheal tube size for children: a radiograph-based formula versus two age-based formulas. J Clin Anesth 2013; 25: 384-7.

11. Shibasaki M, Nakajima Y, Ishii S, Shimizu F, Shime N, Sessler DI. Prediction of pediatric endotracheal tube size by ultrasonography. Anesthesiology 2010; 113: 819-24.

12. Hofer CK, Ganter M, Tucci M, Klaghofer R, Zollinger A. How reliable is length-based determination of body weight and tracheal tube size in the paediatric age group? The Broselow tape reconsidered. Br J Anaesth 2002; 88: 283-5.

13. Wang TK, Wu RS, Chen C, Chang TC, Hseih FS, Tan PP. Endotracheal tube size selection guidelines for Chinese children: prospective study of 533 cases. J Formos Med Assoc 1997; 96: 325-9.

14. Furuya A, Nomura H, Kuroiwa G, Tamaki F, Suzuki S, Nonaka A, et al. Endotracheal tube selection in children: which is the better predictor for the selection, tracheal internal diameter in X-ray photograph or age-based formula? Masui 2009; 58: 724-7.

15. Turkistani A, Abdullah KM, Delvi B, Al-Mazroua KA. The 'best fit' endotracheal tube in children --comparison of four formulae. Middle East J Anaesthesiol 2009; 20: 383-7.

16. Gilsanz V, Ratib O. Hand Bone Age: A digital atlas of skeletal maturity. New York, Springer. 2005, pp 12-3.

17. Kim JR, Lee YS, Yu J. Assessment of bone age in prepubertal healthy Korean children: comparison among the Korean standard bone age chart, Greulich-Pyle method, and Tanner-Whitehouse method. Korean J Radiol 2015; 16: 201-5.

18. Eipe N, Barrowman N, Writer H, Doherty D. A weight-based formula for tracheal tube size in children. Paediatr Anaesth 2009; 19: 343-8.

19. Veyckemans F. Anesthesia equipment. In: Pediatric Anesthesia. Edited by Bissonnette B: Shelton, People's Medical Publishing House-USA. 2011, pp 625-6.

20. Suominen P, Taivainen T, Tuominen N, Voipio V, Wirtavuori K, Hiller A, et al. Optimally fitted tracheal tubes decrease the probability of postextubation adverse events in children undergoing general anesthesia. Paediatr Anaesth 2006; 16: 641-7. 